\title{
ABUNDANCES IN WOLF-RAYET STARS, LBVs and OBN STARS
}

\author{
Allan J Willis \\ Department of Physics \& Astronomy \\ University College London \\ Gower Street \\ London WC1E 6BT, UK
}

\begin{abstract}
Current knowledge of the chemical nature of WR stars determined from $U V$, optical and $I R$ spectral analyses, is reviewed. By number, the $\mathrm{H} / \mathrm{He}$ ratio is low in most WN stars: $10 \geq \mathrm{H} / \mathrm{He} \geq 0.4$ for WNL stars; $3.0 \geq \mathrm{H} / \mathrm{He} \geq 0.0$ for WNE stars with most having near zero $\mathrm{H}$-abundances. The $\mathrm{C} / \mathrm{N}$ ratio in $\mathrm{WN}$ stars lies in the range 1-6 $\times 10^{-2}$, whilst $\mathrm{N} / \mathrm{He} \sim 10^{-4}-4 \times 10^{-3}$. These abundances for WN stars are broadly consistent with equilibrium $C N O$-burning products. In $\mathrm{WC}$ stars $\mathrm{H} / \mathrm{He}=0$ whilst $\mathrm{C} / \mathrm{He} \sim 0.1-0.7$ with some evidence for increasing values from WC7 $\longrightarrow$ WC4. $\mathrm{N}$ is highly deficient in WC stars, with $\mathrm{C} / \mathrm{N} \geq 60$, and for $\mathrm{HD} 165763 \mathrm{O} / \mathrm{C} \leq 0.2$ is deduced. In $\gamma$ Velorum (WC8+O9), IR spectra give a value of $\mathrm{Ne} / \mathrm{He}=1.0 \times 10^{-3}$ (about twice solar). Thus WC abundances are broadly consistent with He-burning products, although the Ne abundance appears lower than predicted. WO stars show $\mathrm{C} / \mathrm{He} \sim 0.2-0.7$, and $\mathrm{O} / \mathrm{He} \sim 0.03-0.1$, revealing both He-burning and $\alpha$-capture products. Ring nebulae around several WN stars shown enhanced $\mathrm{He}$ and $\mathrm{N}$, consistent with mass loss enrichment expectations from their chemically evolved central stars. Enhanced He (and probably $\mathrm{N}$ ) is also seen in the atmospheres of several LBVs, OBN and Of stars - potential precursors of, at least, some WN subtypes.
\end{abstract}

\section{Introduction}

There is now overwhelming evidence that the Pop I WR stars represent the evolved descendents of massive O-type stars, in which extensive mass loss, and/or mass exchange in binary systems, has 'peeled down' the stellar atmospheres to reveal successive products of interior nuclear burning to direct observation (CNO-products in WN stars and He-burning products in WC and WO stars). Herein I will outline results of chemical abundance determinations that have contributed towards this scenario. Previous reviews of this field have been given by Willis (1982a), and Hillier (1990a,b). Progress in overall WR research is reported by Abbott \& Conti (1987) and in the proceedings of IAU Symposia Nos. 99, 116 and 143 (de Loore \& Willis 1982, de Loore, Willis \& Laskarides 1986, van der Hucht \& Hidayat 1990). Additionally I will summarise recent results of observationally inferred abundances in Luminous Blue Variable stars (LBVs) (see proceedings of IAU Colloquium No. 113: Davidson, Moffat \& Lamers 1989) and of OBN stars (see Walborn 1988), since these may have some direct/indirect evolutionary connection with the WR class. 


\section{WR spectral surveys and atlases}

The Pop I WR stars have historically been divided into two sequences: WN stars, whose optical spectra exhibit strong emission lines predominantly in He and $\mathrm{N}$ ions, and WC stars which show emission lines mainly in $\mathrm{He}, \mathrm{C}$ and $\mathrm{O}$. Individual stars in each sequence are ascribed a subtype classification (WN9,...WN3,WN2: WC9,..WC5,WC4) based mainly on ionic line strength ratios (presumed to reflect different ionisation conditions in their winds - see van der Hucht et al. 1981, Abbott \& Conti 1987). Recently a further, WO, sequence has been identified (Barlow \& Hummer 1982) where the spectra show very strong $O$ VI lines in addition to He and C. The past 10-15 years has seen an upsurge in the volume and quality of WR spectroscopy, at $U V$, optical and $I R$ wavelengths, coupled with advances in the development of meaningful model atmosphere techniques, leading to the first reliable quantitative chemical abundance determinations.

Smith \& Kuhi (1981) present an atlas of rectified coude spectral tracings of 13 galactic WN stars covering $\lambda \lambda 3150-7000 \AA$ at $\sim 0.5 \AA$ resolution, including measurements of the optical continuum flux distributions, line equivalent widths and relative fluxes. The subsequent use of electronic, linear detectors has led to compilations of spectrophotometric line measurements of larger samples of WR stars in the Galaxy and Magellanic Clouds. Conti, Leep \& Perry (1983) present line strengths for the He II Pickering lines and the leading NIII, IV, V lines in 72 Galactic WN stars and 33 WN stars in the LMC. Torres, Conti \& Massey (1986) provide equivalent widths of OV $\lambda$ 5590, C III $\lambda 5696$ and C IV $\lambda 5806$ in 65 galactic WC stars and $18 \mathrm{WC}$ stars in the LMC. Conti \& Massey (1989) provide further spectrophotometry of the leading He I, He II, N and C and $\mathrm{O}$ lines in nearly all galactic and LMC WR stars; Conti, Massey \& Garmany (1990) corresponding measurements of the WR stars in the SMC. Further equivalent width measurements of the He spectra of many galactic WN and WC stars are given by Schmutz, Hamann \& Wessolowski (1989).

Conti, Massey \& Vreux (1990) present spectroscopy and line strength measurements of the leading emission features in the near- $I R$ spectra of $47 \mathrm{WN}$ stars and $36 \mathrm{WC}$ stars in the Galaxy. Vreux et al. (1989) present further near-IR spectroscopy covering the region $\lambda \lambda 6150-10350 \AA$ of 5 WN LMC stars, 4 SMC WR stars and 3 galactic WN stars. Smith \& Hummer (1988) present spectral tracings and line equivalent widths and fluxes for leading emission lines in the $I R \mathrm{H}(1.4-1.8 \mu \mathrm{m})$ and $\mathrm{K}(2.0-2.5 \mu \mathrm{m})$ bands of 17 southern galactic WC stars.

In the vacuum ultraviolet $(\lambda \lambda 1150-3250 \AA)$ early low resolution $(\delta \lambda \sim 35 \AA) \mathrm{S} 2 / 68$ spectrophotometry with the TD1-A satellite of 9 galactic WR stars (Willis \& Wilson 1978), has been followed by $I U E$ low resolution $(\delta \lambda \sim 6 \AA)$ spectrophotometry of 15 galactic WR stars (Nussbaumer et al. 1982), and of 9 LMC WR stars (Smith \& Willis 1983), and at high resolution $(\delta \lambda \sim 0.1 \AA)$ of 14 galactic WR stars (Willis et al. 1986).

\section{WR model atmosphere techniques}

The basic picture of a WR star consisting of a hot stellar 'core' surrounded by a dense, rapidly expanding stellar wind producing the emission line spectrum precludes the use of 'normal' stellar atmosphere modelling techniques. WR mass loss rates are very high, $\sim 10^{-5}-10^{-4} \mathrm{M}_{\odot} \mathrm{yr}^{-1}$, (Abbott et al. 1986, Willis 1990), and both continuum and line radiative transfer computations require the use of spherical, NLTE, moving atmospheric modelling for quantitative work - a formidable task.

Simplifications introduced by use of the Sobolev (1960) formulation for spectral line transfer in a rapidly moving wind, were developed by Castor (1970), and subsequently used 
Fig 1: Observed (full lines) He I and He II profiles in HD 96548 (WN8) compared to theoretical profiles from the Kiel group models (Hamann et al. 1990). The $\lambda 4860$ and $\lambda 4340$ profiles determine the $\mathrm{H} / \mathrm{He}$ mass fraction as 0.10 .
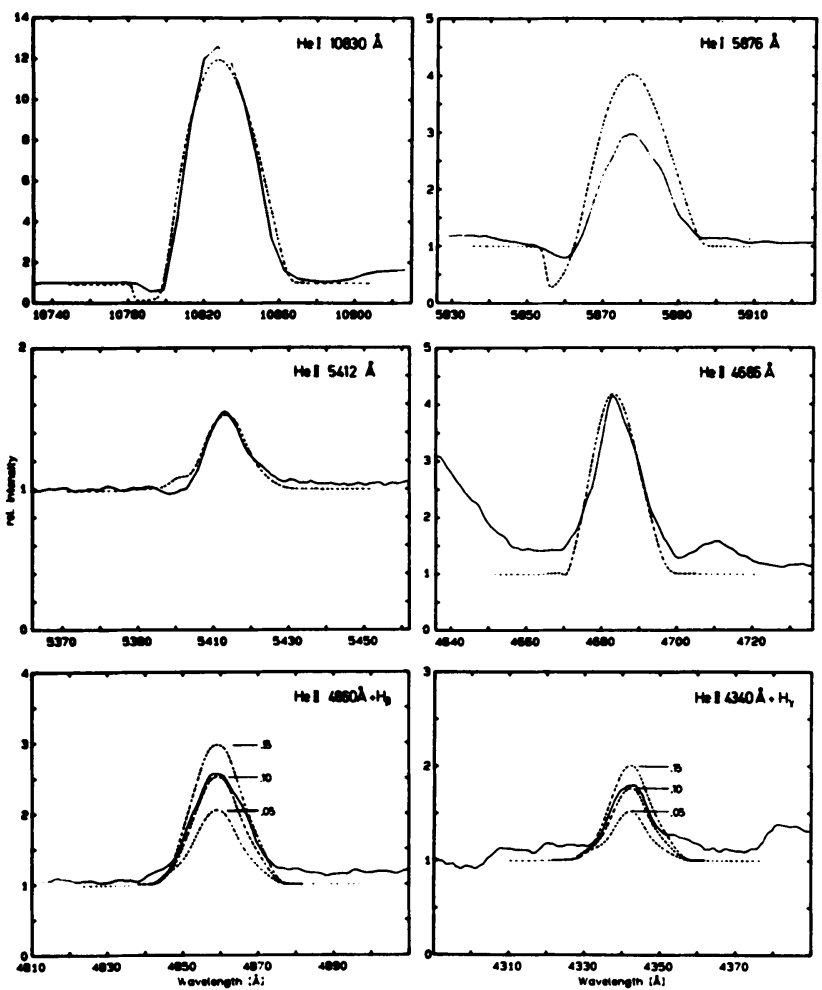

by Castor \& van Blerkom (1970), and Oegerle \& van Blerkom (1976) in their analyses of WR He lines, and Willis \& Wilson (1978), and Smith \& Willis $(1982,1983)$ who estimated the $\mathrm{H}, \mathrm{He}, \mathrm{C}$ and $\mathrm{N}$ abundances in several galactic and LMC WR stars. These studies employed the simplified Escape Probability Method (EPM) for handling line transfer, using a simple 'core-halo' approach, in which the emission line region is specified by a single, 'representative', radius $\left(R_{p}\right.$, velocity $\left.=v_{p}\right)$ surrounding a stellar core of temperature $T_{*}$, and radius $R_{*}$. Multi-level $\mathrm{H}, \mathrm{He}, \mathrm{C}$ and $\mathrm{N}$ ions are used with appropriate $\mathrm{b}-\mathrm{b}$ and b-f populating and de-populating mechanisms to compute individual transition source functions and line strengths for grids of electron density, $\mathrm{N}_{e}$, electron temperature, $\mathrm{T}_{e}$, and ionic species density, $\mathrm{N}_{x}{ }^{+}$, at $\mathrm{R}_{p}$, and compared with observed line strengths in appropriate ions to fix ionic densities and relative abundances.

More recently, these 'one-point' models have been greatly enhanced by the development of simultaneous solutions of the equations of statistical equilibrium, and line and continuum transfer throughout the stellar winds, by workers at JILA (Hillier $1987 \mathrm{a}, \mathrm{b}$ ), and, independently, at Kiel (Hamann \& Schmutz 1987, Hamann, Wessolowski, \& Schmutz 1988, and Schmutz, Hamann \& Wessolowski 1989). Reviews of these new models have been given by Hillier (1990 a,b). They assume: (i) spherical geometry, (ii) a monotonic 
velocity law, $\mathbf{v}(\mathbf{r})=\mathbf{v}_{\infty}\left(1-\mathbf{r}_{c} / \mathbf{r}\right)^{\beta}$, with $\beta=1$ usually adopted; (iii) time-independence, with continuity giving $\dot{M}=4 \pi \mathrm{r}^{2} \rho(\mathrm{r}) \mathrm{v}(\mathrm{r})$; and (iv) homogeneity. Individual models are characterised by a core effective temperature, mass loss rate, wind terminal velocity, core radius $\mathrm{R}_{*}$, and chemical composition. In the Kiel models the wind temperature structure is derived from the assumption of radiative equilibrium computed in the grey LTE approximation, whilst Hillier allows for cooling by the inclusion of metal lines. The bulk of the effort to date in the use of these new models has been in the analysis of the observed WR continuum energy distributions and helium line spectra, to determine basic WR quantities of, $\dot{M}$, luminosity, $\mathrm{T}_{\text {eff }}, \mathrm{R}_{*}$, and the $\mathrm{H} / \mathrm{He}$ ratio - (see Hillier $(1987 \mathrm{a}, \mathrm{b})$ and Hamann, Schmutz \& Wessolowski (1988) for detailed analyses of HD 50896 WN5; Hillier (1989) for HD 167653 WC5; and Schmutz, Hamann \& Wessolowski (1989) for an analysis of the He spectrum of $30 \mathrm{WR}$ stars). Hillier $(1987 \mathrm{~b}, 1989)$ has also derived inferences on the $\mathrm{N}, \mathrm{C}$ and $\mathrm{O}$ abundances. It is now possible to predict and compare theoretical and observed line profiles for the first time. The results are very encouraging (see Fig 1) and confirm the basic reliability of the theory employed. These new models are also able to confirm or refute abundances derived by earlier workers or deduced by the use of more simple or alternative methods of analysis (see below).

\section{Abundances in WN stars}

\subsection{The $\mathrm{H} / \mathrm{He}$ ratio}

The $\mathrm{H} / \mathrm{He}$ ratio is WN stars is most often determined through measurements of the He II Pickering decrement ( a plot of the $\log$ of the observed line intensities, $f_{\lambda}$, of the He II (n-4) series against ' $n$ ') where even-n series members coincide in wavelength with $\mathrm{H}$-Balmer lines (n-2). If appreciable $\mathrm{H}$ is present the Pickering decrement should show an odd-even $n$-bumpiness, with $\mathrm{H} / \mathrm{He} \propto \Delta \mathrm{f}_{\lambda}$. Smith (1973) used this technique to infer low $\mathrm{H} / \mathrm{He}$ ratios for most WN stars observed in the Smith \& Kuhi (1981) spectral atlas, whilst similar conclusions were reached by Willis \& Wilson (1978) and Smith \& Willis $(1982,1983)$. These analyses asserted that high-lying Pickering lines $(\mathbf{n} \geq 10)$ are optically thin - an assumption confirmed in the EPM calculations of Castor \& van Blerkom (1970). This method was extended to a larger sample of galactic WN stars by Conti, Leep \& Perry (1983, CLP) who interpreted observed Pickering decrements in 37 Galactic and $21 \mathrm{LMC}$ WN stars to estimate $\mathrm{H} / \mathrm{He}$ ratios assuming either an optically thin relation: $\log \Delta \mathrm{f}_{\lambda}=\log \left[\left(\mathrm{N}\left(\mathrm{H}^{+}\right) / \mathrm{N}\left(\mathrm{He}^{++}\right)\right)+1\right]$ or an optically thick relation: $\log \Delta \mathrm{f}_{\lambda}=2 / 3 \log \left[\left(\mathrm{N}\left(\mathrm{H}^{+} / \mathrm{N}\left(\mathrm{He}^{++}\right)\right)+1\right]\right.$. Their results are shown graphically in Fig 2 . Nearly all WN stars show substantially reduced $\mathrm{H} / \mathrm{He}$ ratios over the solar value $(\sim 10)$ : WN7 and WN8 stars exhibit the largest values spanning $\sim 0.4-10.0$, whilst WNE stars show little evidence for any $\mathrm{H}$, although a few WNE stars may have $\mathrm{H} / \mathrm{He}$ values as high as 3 . In general, at a given subclass, the stars with the strongest emission line spectra have no detectable hydrogen (NB. most of the data from CLP plotted in Fig 2 , with $\log (\mathrm{H} / \mathrm{He}) \leq 0.0$ are upper limits)

Vreux et al. (1989) have used near-IR measurements of the He II lines and corresponding $\mathrm{H}-\mathrm{Paschen}$ series to derive $\mathrm{H}^{+} / \mathrm{He}^{++}$ratios in 6 galactic $\mathrm{WN}$ and $2 \mathrm{LMC} \mathrm{WN}$ stars, and these results are also shown in Fig 2. In all cases excellent agreement was found with the optically-thin results from CLP.

Bhattia \& Underhill (1986) questioned the reliability of the $\mathrm{H} / \mathrm{He}$ ratios deduced from Pickering decrement analyses, suggesting that insufficient account was being taken of NLTE effects in the complex model atoms required for detailed transfer calculations, as well as possible line-blending effects. They argue that the wind electron temperatures 
Fig 2: $B / H e$ ratios (by number) determined for galactic and LMC WN stars from observed Pickering decrements (CLP), IR spectra (Vreux et al. 1989) and fine line profile analyses (Hamann et al. 1990).
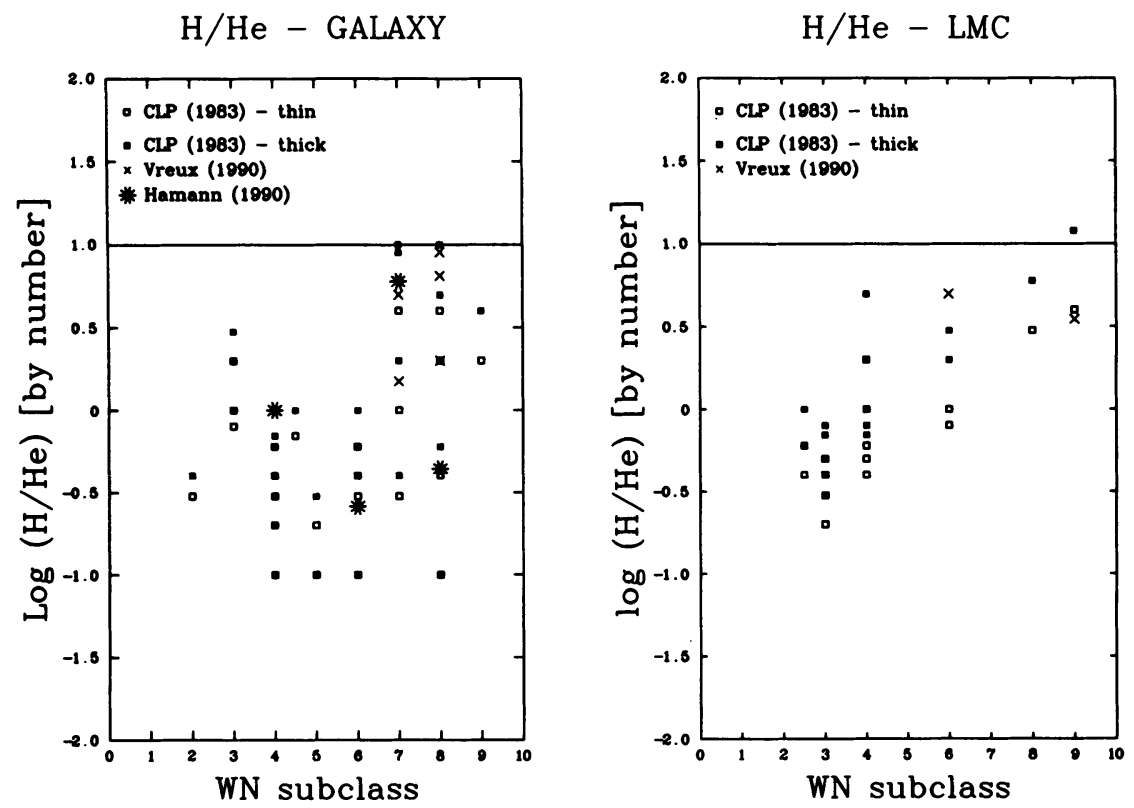

are $\geq 10^{5} \mathrm{~K}$ unless it is assumed that $\mathrm{H} / \mathrm{He}$ is much less than unity. However, Crowther, Smith \& Willis (1990), who use the same complex H and He model atoms in their EPM analysis as employed by Bhatia \& Underhill (1986), are unable to reproduce the latter's results - rather they confirm that the observed Pickering decrements for WN stars imply $\mathrm{H} / \mathrm{He}$ ratios substantially lower than solar, over a very wide range of assumed values of $\mathrm{N}_{e}, \mathrm{~T}_{e}, \mathrm{R}_{*}, \mathrm{~T}_{*}$ in the winds.

Finally, Hamann et al. (1990) have used the new Kiel models (noted above) to examine the $\mathrm{H} / \mathrm{He}$ ratios in $4 \mathrm{WN}$ stars from a fine-analysis of the optical He II line profiles. For individual stars, the stellar parameters of $\mathrm{R}_{*}, \mathrm{~T}_{*}, \dot{M}$, and $\mathrm{v}_{\infty}$, are fixed by line profile fits to unblended He II lines, and by fitting the He II+H I blends the H/He ratio is determined to an accuracy of about $5 \%$ (see Fig 1). Their results are shown in Fig 2 and also show good agreement with the determinations from CLP.

\subsection{CNO abundances}

Willis \& Wilson (1978) used the EPM applied to S2/68 ultraviolet spectrophotometry of emission lines in C III] $\lambda 1909$, C III $\lambda 2296, C$ IV $\lambda 1550$, and isoelectronic transitions of N IV] $\lambda 1486, N$ IV $\lambda 1718$ and NV $\lambda 1240$ to estimate the $C / N$ ratios in 9 galactic WR stars, and this work was superceded by Smith \& Willis $(1982,1983)$ who analysed the same lines in superior IUE UV spectra of 10 galactic and 9 LMC WR stars. The derived $\mathrm{C} / \mathrm{N}$ ratios were considered accurate to a factor of two or so, since isoelectronic 
transitions were compared from low-lying levels in the dominant ionisation stages of these species, with uncertainties arising from the use of the single-point modelling effectively 'cancelling out'. The resulting C/N ratios derived by Smith \& Willis (1983) are reproduced in Fig 3, and show: (i) similar values between galactic and LMC WN counterparts, (ii) $\mathrm{C} / \mathrm{N} \sim 2 \times 10^{-2}-6 \times 10^{-2}$ for WNE stars, and $\sim 6 \times 10^{-3}-4 \times 10^{-2}$ for WNL stars, (iii) values considerably smaller than the solar $\mathrm{C} / \mathrm{N}$ ratio $\sim 3$, (iv) similarity to the equilibrium CNO nuclear burning value of $\sim 2 \times 10^{-2}$ (eg. Maeder 1983, Maeder \& Meynet 1987, Prantzos et al. 1986). The deduced C/He and N/He ratio implied a combination of C-depletion and $\mathrm{N}$-enhancement, but the $\mathrm{N} / \mathrm{He}$ values deduced were a factor of $\sim 7$ lower than predicted in the evolutionary models. Smith \& Willis (1983) argued that this difference was probably not significant given the crudeness of the modelling, and the different line formation processes operating in producing the $\mathrm{C}-\mathrm{N}$ and He lines studied.

Nugis (1982, including references to his earlier work) has analysed the optical spectra published by Smith \& Kuhi (1981) of 5 WN stars to derive N/He $0.002-0.02$ by number, spanning the theoretical CNO-equilibrium value of $\sim 4 \times 10^{-3}$.

For HD 50896 (WN5), Hillier (1987a,b) carried out a very detailed NLTE model atmosphere analysis to determine the stellar parameters $\dot{M}, \mathrm{~T}_{\text {eff }}$ and $\mathrm{R}_{*}$. Subsequently Hillier (1988) used these results to model observed optical and $U V \mathrm{He}, \mathrm{C}, \mathrm{N}$ and $\mathrm{O}$ lines to determine their abundances. His values gave: $\mathrm{C} / \mathrm{N}=0.07$ - very close to the result reported by Smith \& Willis $(1982)-\mathrm{N} / \mathrm{He} \sim 10^{-4}-4 \times 10^{-3}$, with the upper value being consistent with CNO-burning expectations. Hamann (1990) reports the first results from $\mathrm{N}$-line analyses using the Kiel models, and for WN stars finds $\mathrm{X}(\mathrm{N})_{\text {mass }}=0.015$, in good agreement with previous determinations above. Although few suitable O-lines were available for study, Hillier (1988) found an upper limit of $\mathrm{O} / \mathrm{N} \leq 3$ for HD 50896, of limited use in constraining theory which predicts a value of $\sim 0.04$.

On balance the $\mathrm{H}, \mathrm{He}, \mathrm{C} \& \mathrm{~N}$ abundances currently derived from $U V$-optical spectra of WN stars are broadly consistent with theoretical $C N O$-burning equilibrium values - with these interior processed products being exposed to observation via previous atmospheric stripping by extensive mass loss.

\section{Abundances in WN-C stars}

Three galactic stars in the van der Hucht et al. (1981) catalogue are classified as having both WN and WC optical spectral characteristics, and Conti \& Massey (1989) have suggested 6-7 galactic WN-C candidates and 2 in the LMC on the basis of anomalous C IV $\lambda 5800 / \mathrm{He}$ II $\lambda 4686$ line strengths in their survey of 'WN' spectra. To date only the brightest of these objects, HD 62910 (WN6-C4) has been subjected to a detailed analysis. Willis \& Stickland (1990) have used the one-point EPM method to analyse its optical and IUE UV He, $\mathrm{C}$ and $\mathrm{N}$ spectrum, yielding the following mass abundance estimates: $\mathrm{C} / \mathrm{He} \sim$ $2 \times 10^{-3} ; \mathrm{N} / \mathrm{He} \sim 1 \times 10^{-2} ; \mathrm{C} / \mathrm{N} \sim 0.3$ and $\mathrm{H} / \mathrm{He}=0.0$. These values are intermediate between those determined, using similar techniques, for WNE and WC stars (see below), and it is concluded that HD 62910 is a single WR star on its way from a WN6 stage to a WCE phase. Conti \& Massey (1989) suggest that, like HD 62910, most WN-C stars in their sample are single objects, and it is highly plausible that they all represent such intermediately evolved stars. In that case, available statistics would suggest a lifetime ratio $t_{W N-C} / t_{W R} \sim 4 \%$ - considerably longer than predicted in the evolutionary models of Maeder \& Meynet (1987), although Langer's (1990) models do predict a significant WN-C phase. 
Fig 3: $C / N$ ratios (by mass) in WN stars derived from EPM analyses by Smith $\&$ Willis (1982, 1983)

Fig 4: The C/He ratio (by number) derived by Smith $\&$ Hummer (1988) for WC stars based on infrared $H, K$ spectroscopy.
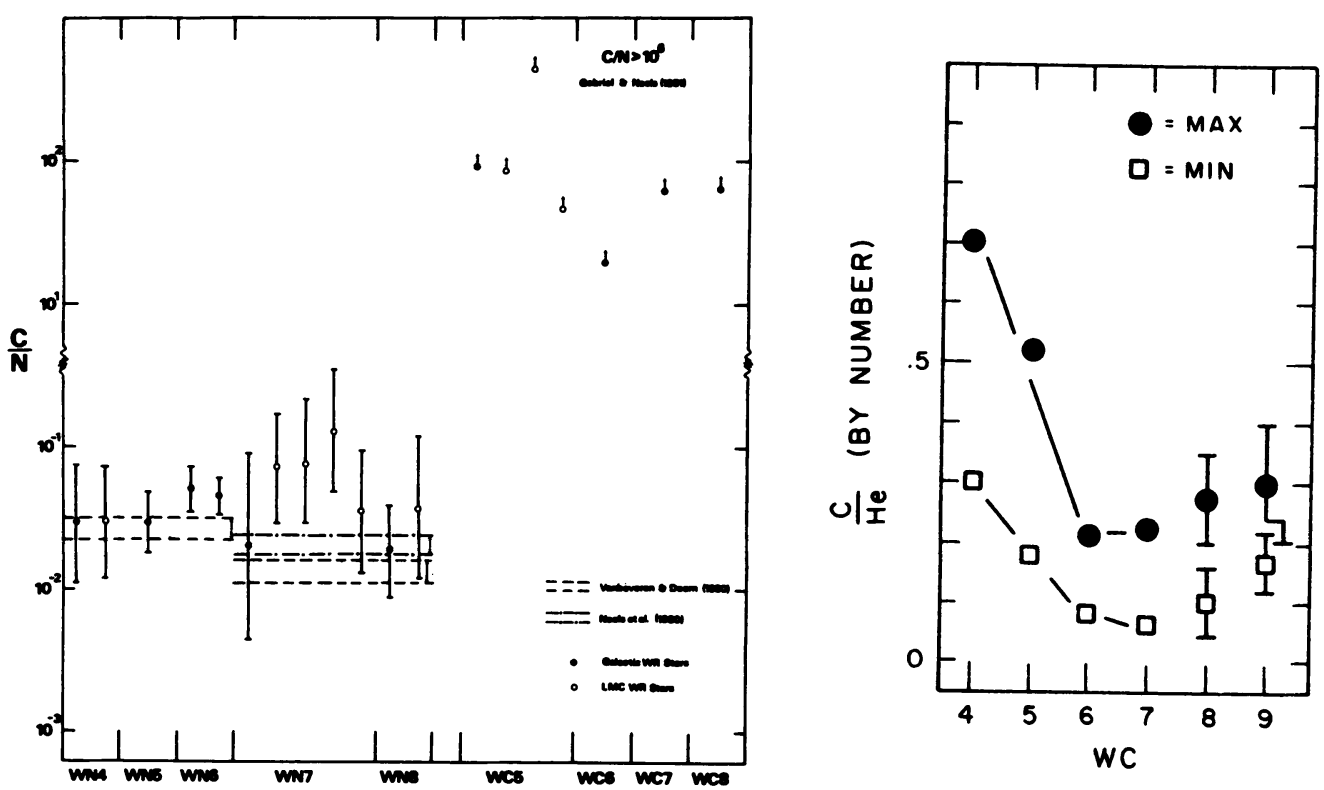

\section{Abundances in WC stars}

\subsection{The $\mathrm{H} / \mathrm{He}$ ratio}

$\mathrm{A} \mathrm{H} / \mathrm{He}$ ratio $=0$ is generally adopted for $\mathrm{WC}$ stars based on the fact that no unambiguous identification of hydrogen lines have been made in their optical or IR spectra. Severe blending of optical He II lines with neighbouring $\mathrm{C}$ and $\mathrm{O}$ transitions precludes the use of the He II Pickering decrement, with Nugis (1975) setting an upper limit of $\mathrm{H} / \mathrm{He} \leq \mathbf{0 . 1}$. Willis (1982b) found no evidence in IUE WC spectra for P Cyg absorption in $H$ Lyman- $\alpha$ supporting an effective zero $H$-abundance. Stellar evolution theory, in which WC stars are further evolved from WN stars (eg. Maeder 1983), predicts $\mathrm{H} / \mathrm{He}=0.0$, and at this time it is probably safe to assume that WC stars are devoid of hydrogen.

\subsection{CNO abundances}

Smith \& Willis $(1982,1983)$ used the EPM model to analyse the IUE UV spectra of 4 galactic and 3 LMC WC stars using the C III] $\lambda 1909, C$ III $\lambda 2296, C$ IV $\lambda 1550$, He II $\lambda 1640$ and He II (n-3) Fowler series, to derive $\mathrm{C} / \mathrm{He} \sim 0.003-0.01$, considerably lower than predicted for He-burning products in the evolutionary models (Maeder 1983), viz 0.1-0.7. Analyses of optical $C$ and He lines in WC spectra by Nugis (1982b) gave values of $\mathrm{C} / \mathrm{He} \sim 0.1-0.2$, more in line with evolutionary expectations, whilst Torres (1988) 
found values of $\mathrm{C} / \mathrm{He} \sim 0.1-0.8$ from a simple recombination treatment of optical $\mathrm{C}$ and He emission lines .

Smith \& Hummer (1988) have analysed the near- IR $\mathrm{H}$ and $\mathrm{K}$ spectra of 17 southern WC stars using recombination theory with allowance for collisional redistribution for observed transitions in He I, He II and C IV. Observed lines in C III and C II (for which such atomic calculations were not available) had to be treated with LTE, with subsequent assessments of the maximum NLTE effects expected, providing upper estimates to the $\mathrm{C} / \mathrm{He}$ ratio. They found $\mathrm{C} / \mathrm{He} \sim 0.04-0.3$ by number (with C II,III in LTE) rising to $\mathrm{C} / \mathrm{He} \sim 0.1-0.7$ when NLTE effects were considered. The $\mathrm{C} / \mathrm{He}$ ratio was found to decrease from WC4 to WC7 (see Fig 4).

Hillier (1989) has employed his detailed model atmosphere codes to study the $U V$ and optical spectrum of $\mathrm{HD} 165763$ (WC5), finding a value of $\mathrm{C} / \mathrm{He} \sim 0.5$. He shows convincingly that the reason for past discrepancies between the above $U V$ and optical results lies in the omission in the $U V$ treatment of dielectronic recombination in the formation of lines like C III $\lambda 2296$, and consequent overestimates of the wind electron temperature. Hamann (1990) using the new Kiel models to analyse WC spectra derives $\mathrm{X}(\mathrm{C})_{\text {mass }}=0.5$. Hillier (1989) also sets a rough upper limit to $\mathrm{O} / \mathrm{C} \leq 0.2$ in HD 165765 using the optically thin transition OIII $\lambda 3625$ formed by recombination. This value is somewhat lower than predicted in the models of Maeder (1983).

To my knowledge there is currently no unambiguous identification of nitrogen in WC spectra (weak optical $\mathrm{N}$ emissions have been reported from time to time, eg. Bappu (1973) but never substantiated; whilst past identifications of N IV]1486, N IV $\lambda 1718$ in S2/68 spectra (Willis \& Wilson 1978) have been negated by better quality IUE spectra (Willis 1980, Smith \& Willis 1982, 1983). Setting lower limits of $W_{\lambda} \leq 1 \AA$ on the $U V$ N IV lines, Smith \& Willis $(1982,1983)$ deduced a lower limit of $C / N \geq 60$ for WC stars (see Fig 3). Evolutionary models predict a very low $\mathrm{N}$-abundance in WC stars, ie. $\mathrm{N} / \mathrm{He} \leq$ $10^{-7}$ (Prantzos et al.. 1986, Maeder 1983) consistent with the lack of observable nitrogen.

\subsection{Neon abundance}

Van der Hucht \& Olnon (1985) observed the [Ne II] $\lambda 12.8 \mu m$ and [Ne III] $\lambda 15.5 \mu m$ emission features in the IRAS low resolution spectrum of $\gamma$ Velorum (WC8+O9I) and deduced a ratio $\mathrm{Ne} / \mathrm{He}=0.009$ using simple recombination theory $-\mathrm{a}$ value $\sim \times 10$ solar and close to that predicted in evolutionary theory for He-burning products (Maeder 1983), in which substantial $\mathrm{Ne}^{22}$ is produced.

However, Barlow, Roche \& Aitken (1988) secured superior resolution $(\mathrm{R}=330) I R$ spectra of $\gamma$ Velorum, resolving the [Ne II] $12.8 \mu \mathrm{m}$ line and rederived the neon abundance using improved analysis techniques. Their result gave $\mathrm{Ne} / \mathrm{He}=1.0 \times 10^{-3}$, only $\sim \times 2$ solar, is to be preferred over the previous estimate and suggests that substantial $\mathrm{Ne}^{22}$ is not present in WC stars, a result which presents a serious challenge to chemical evolution models.

\section{Abundances in WO stars}

Following the identification by Sanduleak (1971) of 5 stars with very strong O VI $\lambda 3811$ emission but not apparently associated with planetary nebulae, Barlow \& Hummer (1982) secured optical and $I U E$ spectra of these objects, confirmed their high ionisation state and, for Sand 1, 2, 4 and 5 proposed a designation as Pop I WO-type stars. For Sand 3 (which they considered a remnant star of a PN with the nebulae no longer visible) the observed $\mathrm{He}, \mathrm{C}$ and $\mathrm{O}$ emission line spectrum compared with Case $\mathrm{B}$ recombination theory, yielded 
the following mass abundances: $\mathrm{H}: \mathrm{He}: \mathrm{C}: \mathrm{O}=0: 38: 54: 8$, implying substantial enhancement of $\mathrm{O}$ through $\alpha$-particle capture on $\mathrm{C}$, in addition to triple- $\alpha$ processing of He to $\mathrm{C}$. The IUE spectrum of Sand 3 was noted to also have strong $\mathrm{Ne} \mathrm{V}$ (and possibly $\mathrm{Ne} \mathrm{VI}$, and $\mathrm{Ne}$ VII) consistent with $\alpha$-particle capture on $O$.

Very recently, Kingsburgh \& Barlow (1990) have determined the properties of the Pop I WO stars Sand $1,2,4$ and 5 , and derived the $\mathrm{C}^{4+} / \mathrm{He}^{2+}$ and $\mathrm{O}^{6+} / \mathrm{He}^{2+} \mathrm{ra}$ tios using the recombination technique of Barlow \& Hummer (1982). Their results for $\mathrm{C}^{4+} / \mathrm{He}^{2+}:: \mathrm{O}^{6+} / \mathrm{He}^{2+}$ lie in the range $0.20:: 0.03$ (Sand 5 , WO2), through $0.38:: 0.03$ (Sand 2, WO4), 0.51::0.08 (Sand 1, WO4+O7) to 0.66::0.10 (Sand 4, WO1), and point very clearly to WO stars being further evolved from WC stars, with both triple- $\alpha$ processing and $\alpha$-capture products being observed.

\section{Inferences from WR ring nebulae}

A substantial number of WR stars (particularly WN) are surrounded by ring nebulae of different morphological types (see Chu et al. 1983). Recent high signal-to-noise, spatially resolved and extended wavelength coverage optical spectra of many of these nebulae, coupled to the use of improved central star photoionising model atmospheres (e.g. Hamann \& Schmutz 1987, Wessolowski et al. 1988), have led to accurate determinations of nebulae $\mathrm{H}, \mathrm{He}, \mathrm{N}$ and $\mathrm{O}$ abundances, revealing important information on the extent of chemical enrichment from material expelled from the central WR star. Rosa \& Mathis (1990) have studied 5 ring nebulae finding $\mathrm{He}$ and $\mathrm{N}$ enrichments by factors of up to 3 and 10 respectively. Vilchez \& Esteban (1990) in their study of 8 ring nebulae find they can separate $\mathrm{O} / \mathrm{H}$-normal and $\mathrm{O} / \mathrm{H}$-poor objects, where for the latter there is a clear correlation of increasing $\mathrm{N} / \mathrm{O}$ with $\mathrm{O}$-deficiency, with all such objects exhibiting $\mathrm{He}$ enhancements with $\mathrm{Y} \geq 0.35$. Their result implies a $\mathrm{N} / \mathrm{H}$ overabundance of at least $0.8 \mathrm{dex}$ for the O-poor nebulae. Vilchez \& Esteban (1990) conclude that their results point very clearly to processed material from the central WR star being present in many of the ring nebulae. They compare quantitatively, the observed nebulae mass fractions of $N / O$ and $Y$ with the predictions of enrichments from stellar evolution models incorporating mass loss from the M-S up to the WN phase (e.g. Maeder 1990), showing that the observed $N$ and He enhancements broadly agree with the model predictions. These results are providing beautiful, independent confirmation that nuclear processed material is indeed exposed to observation during the evolution of massive stars up to and including the WR phase.

\section{Abundances in Luminous Blue Variable Stars}

Some of the most luminous stars in the Galaxy, MCs and M33 are located in the HR diagram close to the Humphreys-Davidson (1979) limit where the onset of an instability precludes further redward evolution, initiates significant variability and the ejection of substantial atmospheric material. Examples are P Cygni, AG Carinae, Eta Carinae, the Hubble Sandage variables and S Doradus. The physics of Luminous Blue Variable stars has recently been the subject of IAU Colloquium No. 113 (Davidson, Moffat \& Lamers, eds., 1989) and it considered that they may well represent an immediate precursor stage to the WN phase. Evidence for abundance anomalies (viz $\mathrm{He}$ and $\mathrm{N}$ enhancements, and/or $\mathrm{C}, \mathrm{O}$ depletions) comes from both qualitative assessments of their optical and $U V$ spectra (see Walborn 1988), and from optical emission line studies of their surrounding nebulae (presumed to have been ejected at the H-D limit ). Leitherer \& Zickgraf (1987) found a high $[\mathrm{NII}] / \mathrm{H} \alpha$ ratio in the circumstellar shell of $\mathrm{P}$ Cyg, whilst Walborn (1982) found 
enhanced $N$ and depleted $O$ in the shell surrounding R127 (=HDE 269858) in the LMC. An analysis of expanding knots of material in the outer shell of Eta Carinae has revealed He and $N$ enhancements (Davidson, Walborn \& Gull 1982, and Davidson et al. 1986), whilst Allen, Jones \& Hyland (1985) have confirmed the He-overabundance through IR spectroscopy. Enhanced N has been proposed in the LMC and SMC LBVs HD 38489 and S18 by Shore \& Sanduleak (1983) and Shore, Sanduleak \& Allen (1987). A detailed analysis of the $U V$-optical-IR spectrum of P Cyg by Deacon \& Barlow (1990) has yielded a very high ratio of $\mathrm{He} / \mathrm{H}=0.5$.

Much attention is now being focussed on stars now classified as Ofpe/WN9 (the 'Slash-stars', see Walborn 1982, and Bohannan 1990), which show both high excitation Of-lines and low-excitation P Cyg features. It is becoming clear that such stars also show significant abundances anomalies, e.g. Schmutz et al. (1990) have carried out a detailed NLTE analysis of HDE 269227 (=R84) in the LMC, finding a high helium abundance of $\mathrm{Y}=\mathbf{0 . 6 3}$ by mass.

\section{Abundances in OBN stars}

Many OB stars are known which show abnormally strong optical $\mathrm{N}$ lines (the OBN stars) compared to 'normal' OB spectra (see Walborn (1976) for a review of their spectroscopic properties), and recent NLTE modelling has confirmed the significant abundance enhancements that were inferred qualitatively before. (NB. OBC stars are now believed to be essentially 'unevolved' exhibiting natal atmospheric abundances - see Walborn 1988). Schonberner et al. (1987) have performed a differential NLTE analysis of 4 OBN and 2 normal OB stars, finding essentially solar composition in the latter, but enhanced $\mathrm{He}(0.15$ - 0.26 by number), N-enrichment, C-depletion, but O-normal in the OBN stars. Their results point to incompletely $C N O$-cycled material (viz products of CN equilibrium alone) exposed to observation by internal mixing and/or mass loss.

Bohannan et al. (1986) carried out a detailed NLTE analysis of the optical spectrum of $\zeta$ Puppis (O4f), including the effects of wind-blanketing, finding an overabundance of He by $\times 2$ solar. Subsequently, Voels et al. (1989) have completed a parallel analysis of 4 09.5 stars forming a luminosity sequence $\mathrm{Ia}, \mathrm{Ib}, \mathrm{II}, \mathrm{V}$, and find that the most luminous member of the sample ( $\alpha \mathrm{Cam}, 09.5 \mathrm{Ia})$ has enhanced He at 0.18 by number. They argue that He-enrichment, and probably CNO processed material, is likely to be a common feature of stars with OIa and Of classifications. Similarly, Kudritzki et al. (1987) found substantial overabundances of He and $N$ in the LMC stars SK $-65^{\circ} 21$ (O9.5Ia) and SK $-68^{\circ} 41$ (BO.5Ia).

\section{Conclusions and evolutionary implications}

The chemically evolved nature of the WR stars determined by recombination analyses of optical and $I R$ lines and one-point EPM analyses is being confirmed by more recent full NLTE continuum and line transfer modelling. WN stars appear to exhibit products of nuclear CNO-burning, whilst WC and WO stars products of He-burning and alphacapture processes. The possible precursors of WN stars, viz Of, (possibly OBN), and LBVs also show evidence for CNO-processed material. In chemical terms, a potential evolution scheme could be:

$$
\mathrm{O} \longrightarrow \mathrm{Of} \longrightarrow \mathrm{LBV} \longrightarrow \mathrm{Of} / \mathrm{WN} \longrightarrow \mathrm{WN}(\mathrm{L}, \mathrm{E}) \longrightarrow \mathrm{WC} \longrightarrow \mathrm{WO}
$$

For an individual initial mass $O$ star, not all elements of this sequence may be reached. 
The exposition of interior nuclear-processed material is via extensive mass loss (prior to and at the WR stages), possibly assisted in the case of binary systems by mass transfer. WR abundances (converted to mass fractions) derived from spectral analyses are compared below with predictions of single star evolution models from Maeder \& Meynet (1987).

\begin{tabular}{lll}
\hline \multicolumn{1}{c}{ Theory (Maeder \& Meynet 1987) } & Observed values & agreement \\
\hline WN: & & \\
$\mathrm{C} / \mathrm{He}=(1.8-3.9) \times 10^{-4}$ & $2.1 \times 10^{-5}-8.4 \times 10^{-4}$ & yes \\
$\mathrm{N} / \mathrm{He}=(1.3-1.7) \times 10^{-2}$ & $3.5 \times 10^{-4}-1.4 \times 10^{-2}$ & yes \\
$\mathrm{C} / \mathrm{N}=(1.1-2.9) \times 10^{-2}$ & $(0.6-6.0) \times 10^{-2}$ & yes \\
$\mathrm{C} / \mathrm{O}=0.25-1.3$ & $\geq 0.02$ & - \\
$\mathrm{WC}-\mathrm{WO}:$ & & \\
$\mathrm{C} / \mathrm{He}=0.2-3.0$ & $0.12-2.0$ & yes \\
$\mathrm{O} / \mathrm{C}=0.3-2.0$ & $\leq 0.3(\mathrm{WC})-0.2(\mathrm{WO})$ & yes (?) \\
$\mathrm{Ne} / \mathrm{He}=0.03-0.17$ & $0.005(\gamma \mathrm{Vel})$ & No \\
\hline
\end{tabular}

For WN stars the agreement between 'observations' and theory is really very good, given the uncertainties of $\pm \times 2$ or so in the former. It would be good to have an accurate WN O/He measurement. For the WC and WO stars, the $\mathrm{C} / \mathrm{He}$ comparison is satisfactory, the observationally derived $\mathrm{O} / \mathrm{C}$ value appears somewhat low (increasing overshooting may give better agreement - Prantzos et al. 1986), but the $\mathrm{Ne} / \mathrm{He}$ ratio is a factor of $\sim 10$ lower than predicted. In $\S 5$ it was noted that the Maeder \& Meynet (1987) models predict few, if any WN-C stars, because at the onset of He-burning $\mathrm{N}$ is very quickly destroyed through the process ${ }^{14} \mathrm{~N}(\alpha, \gamma){ }^{18} \mathrm{~F}(\beta, \nu)^{18} \mathrm{O}(\alpha, \gamma)^{22} \mathrm{Ne}(\alpha, \mathrm{n})^{25} \mathrm{Mg}$ and it is this chain that leads to the prediction of a high ${ }^{22} \mathrm{Ne}$ abundance in WC stars. The low Ne-abundance found by Barlow, Roche \& Aitken (1988) for $\gamma$ Velorum (WC8+O9I), if applicable to WC stars in general, coupled to the probability of a significant WN-C phase, might suggest that something is wrong with the formulation of this latter process. Maeder (these proceedings) will provide an update on WR evolution models.

\section{References}

Abbott, D.C., Bieging, J.H., Churchwell, E., \& Torres, A.V., 1986, Astrophys. J., 303, 239

Abbott, D.C., \& Conti,P.S., 1987, Ann. Rev. Ast. Astrophys., 25, 113

Allen, D.R., Jones, T.J., \& Hyland, A.R., 1985, Astrophys. J., 291, 280

Bappu, M.K.V., 1973, Proc. IAU Symp. No. 49, (eds M K V Bappu \& J Sahade), D. Reidel, p 59

Barlow, M..J., \& Hummer, D.G., 1982, Proc IAU Symp. No. 99, (eds C de Loore \& A J Willis), D. Reidel, p 387

Barlow, M.J., Roche, P.F., \& Aitken, D.K., 1988, Mon. Not. R. astr. Soc., 232, 821 Bhattia, A.K., \& Underhill, A.B., 1986, Astrophys. J. Suppl., 60, 323

Bohannan, B., 1990, in Properties of Hot Luminous Stars, (ed. C D Garmany), P.A.S.P. Conf. Ser., 7, p 39

Bohannan, B., Abbott, D.C., Voels, S.A., \& Hummer, D.G., 1986, Astrophys. J., 308, 728

Castor, J.I., 1970, Mon. Not. R. astr. Soc., 149, 111 
Castor, J.I., \& van Blerkom, D., 1970, Astrophys. J., 161, 485

Chu, Y.-H., Gull, T.R., Treffers, R.R., Kwitter, K.B., \& Troland, T.H., 1983, Astrophys. J., 254, 562

Conti, P.S., Leep, E.M., \& Perry, D., 1983, Astrophys. J., 268, 228

Conti, P.S., \& Massey, P., 1989, Astrophys. J., 337, 251

Conti, P.S., Massey, P, \& Garmany, C.D., 1990, Astrophys. J., 341, 113

Conti, P.S., Massey, P., \& Vreux, J-M., 1990, Astrophys. J., 354, 359

Crowther, P., Smith, L.J., \& Willis, A.J., 1990, in Proc. IAU Symp. No. 143, (eds. K A van der Hucht \& B Hidayat), Kluwer Acad. Pub., in press

Davidson, K., Walborn, N.R., \& Gull, T.R., 1982, Astrophys. J., 254, L47

Davidson, K., Dufour, R.J., Walborn, N.R., \& Gull, T.R., 1986, Astrophys. J., 305, 867

Davidson, K., Moffat, A.F.J., \& Lamers, H., (eds), 1989, Proc IAU Coll. No. 113, (Physics of Luminous Blue Variables), Kluwer Acad. Pub.

Deacon, J.R., \& Barlow, M.J., 1990, in Proc. IAU Symp. No.143, (eds K A van der Hucht \& B Hidayat), Kluwer Acad. Pub., in press

de Loore, C, \& Willis, A.J., (eds.), 1982, Proc IAU Symp. No 99 (WR stars - observations, physics $\mathcal{G}$ evolution), D. Reidel Pub. Co.

de Loore, C., Willis, A.J., \& Laskarides, P., (eds.), 1986, Proc. IAU Symp. No. 116 (Luminous stars $\&$ associations in galaxies), D. Reidel Pub. Co.

Hamann, W-R., 1990, in Proc. IAU Symp. No. 143, (eds. K A van der Hucht \& B Hidayat), Kluwer Acad. Pub., in press

Hamann, W-R., \& Schmutz, W., 1987, Astr. Astrophys., 174, 173

Hamann, W-R., Schmutz, W., \& Wessolowski, U., 1988, Astr. Astrophys., 194, 190

Hamann, W-R., \& Wessolowski, U., 1990, Astrophys. J., 227, 171

Hamann, W-R., Wessolowski, U., Schwarz, E., Dunnebeil, G., \& Schmutz, W., 1990, in Properties of Hot Luminous stars, (ed. C D Garmany), P.A.S.P. Conf. Ser., 7, p 259

Hillier, D.J., 1987a, Astrophys. J. Suppl., 63, 947

Hillier, D.J., 1987b, Astrophys. J. Suppl., 63, 965

Hillier, D.J., 1988, Astrophys. J., 327, 822

Hillier, D.J., 1989, Astrophys. J., 347, 392

Hillier, D.J., 1990a, in Properties of Hot Luminous stars, (ed C D Garmany), P.A.S.P. Conf. Ser., 7, p 340

Hillier, D.J., 1990b, Proc IAU Symp. No. 143, (eds K A van der Hucht \& B Hidayat), Kluwer Acad. Pub., in press

Humphreys, R.M., \& Davidson, K., 1979, Astrophys. J., 232, 409

Kingsburgh, R., \& Barlow, M.J., 1990, in Proc IAU Symp. No. 143, (eds K A van der Hucht \& B Hidayat), Kluwer Acad. Pub., in press

Kudritzki, R.P., Groth, H.G., Butler, K., Husfeld, D., Becker, S., Eber, F., \& Fitzpatrick, E., 1987, ESO SN 1987A Workshop,

Langer, N., 1990, in Properties of Hot Luminous stars, (ed. C D Garmany), P.A.S.P. Conf. Ser., 7, p 328

Leitherer, C., \& Zickgraf, F.-J., 1987, Astr. Astrophys., 175, 208

Maeder, A., 1983, Astr. Astrophys., 120, 113

Maeder, A., 1990, Astr. Astrophys. Suppl., 84, 139

Maeder, A., \& Meynet, G., 1987, Astr. Astrophys., 182, 243

Nugis, T., 1975, Proc IAU Symp. No. 67, (eds V E Sherwood \& P Lukas), D. Reidel, p 291

Nugis, T., 1982a, Proc IAU Symp. No. 99, (eds. C de Loore \& A J Willis), D. Reidel., p 127 
Nugis, T., 1982b, Proc IAU Symp. No. 99, (eds C de Loore \& A J Willis), D. Reidel, p 131

Nussbaumer, H., Schmutz, W., Smith, L.J., \& Willis, A.J., 1982, Astr. Astrophys. Suppl., 47, 257

Oegerle, W.R., \& van Blerkom, D., 1976, Astrophys. J., 206, 150

Prantzos, N., Doom, C., Arnould, M., \& de Loore, C., 1986, Astrophys. J., 304, 695

Rosa, M.R., \& Mathis, J.S., 1990, in Properties of Hot Luminous Stars, (ed C D Garmany), P.A.S.P. Conf. Ser., 7, p 135

Sanduleak, N., 1971, Astrophys. J., 164, L71

Schmutz, W., Hamann, W-R., \& Wessolowski, U., 1989, Astr. Astrophys., 210, 236

Schmutz, W., Leitherer, C., Hubeny, I., Vogel, M., Hamann, W-R., \& Wessolowski, U., 1990, Astr. Astrophys., submitted

Schonberner, D., Herrero, A., Becker, S., Eber, F., Butler, K., Kudritzki, R.P., \& Simon, K.P., 1988, Astr. Astrophys., 197, 209

Shore, S.N., \& Sanduleak, N., 1983, Astrophys. J., 273, 177

Shore, S.N., Sanduleak, N., \& Allen, D.A., 1987, Astr. Astrophys., 176, 59

Smith, L.F., 1973, Proc IAU Symp. No. 49, (eds M K V Bappu \& J Sahade), D. Reidel, p 15

Smith, L.F., \& Kuhi, L.V., 1981, An atlas of WR line profiles, JILA Report No. 117

Smith, L.F., \& Hummer, D.G., 1988, Mon. Not. R. astr. Soc., 230, 511

Smith, L.J., \& Willis, A.J., 1982, Mon. Not. R. astr. Soc., 201, 451

Smith, L.J., \& Willis, A.J., 1983, Astr. Astrophys. Suppl., 54, 229

Sobolev, V.V., 1960, The moving envelopes of stars, Harvard Univ. Press

Torres, A.V., 1988, Astrophys. J., 325, 759

Torres, A.V., Conti, P.S., \& Massey, P., 1986, Astrophys. J., 300, 379

van der Hucht, K.A., Conti, P.S., Lundstrom, I., \& Stenholm, B., 1981, Spac. Sci. Rev., 28, 227

van der Hucht,, K.A., \& Olnon, 1985, Astr. Astrophys., 149, L17

van der Hucht, K.A., \& Hidayat, B., (eds.), 1990, Proc IAU Symp. No. 143 (WR stars and interrelations with other massive stars in galaxies), Kluwer Acad. Pub.

Vilchez, J.M., \& Esteban, C., 1990, Proc. IAU Symp. No. 143, (eds K A van der Hucht \& B Hidayat), Kluwer Acad. Pub. in press

Voels, S.A., Bohannan, B., Abbott, D.C., \& Hummer, D.G., 1989, Astrophys. J., 340, 1073

Vreux, J-M., Dennefeld, M., Andrillat, Y., \& Rochowich, K., 1989, Astr. Astrophys. Suppl., 226, 421

Walborn, N.R.., 1976, Astrophys. J., 205, 419

Walborn, N.R., 1982, Astrophys. J., 256, 452

Walborn, N.R., 1988, Proc. IAU Coll. No. 108, (ed. K Nomoto), Springer-Verlag Pub., p 70

Wessolowski, U., Schmutz, W., \& Hamann, W-R., 1988, Astr. Astrophys., 194, 160

Willis, A.J., 1980, Proc. 2nd European IUE Conf., ESA Sp-157, p il

Willis, A.J., 1982a, Proc IAU Symp. No. 99, (eds C de Loore \& A J Willis), D. Reidel, p 87

Willis, A.J., 1982b, Mon. Not. R. astr. Soc., 198, 897

Willis, A.J., 1990, in Proc. IAU Symp. No. 143, (eds. K A van der Hucht \& B Hidayat), Kluwer Acad. Pub., in press

Willis, A.J., \& Wilson, R., 1978, Mon. Not. R. astr. Soc., 182, 559

Willis, A.J., van der Hucht, K.A., Conti, P.S., \& Garmany, C.D., 1986, Astr. Astrophys. Suppl., 63, 417

Willis, A.J., \& Stickland, D.J., 1990, Astr. Astrophys., 232, 89 